\title{
Novel perturbation approach for the structure factor of the attractive hard-core Yukawa fluid
}

Andrij Trokhymchuk

Roman Melnyk

Filip Moucka

Ivo Nezbeda

Follow this and additional works at: https://scholarsarchive.byu.edu/facpub

Part of the Biochemistry Commons, and the Chemistry Commons

\section{Original Publication Citation}

Melnyk, Roman, Filip Mou, Ivo Nezbeda, and Andrij Trokhymchuk."Novel perturbation approach for the structure factor of the attractive hard-core Yukawa fluid." The Journal of Chemical Physics 127 (27).

\section{BYU ScholarsArchive Citation}

Trokhymchuk, Andrij; Melnyk, Roman; Moucka, Filip; and Nezbeda, Ivo, "Novel perturbation approach for the structure factor of the attractive hard-core Yukawa fluid" (2007). Faculty Publications. 234.

https://scholarsarchive.byu.edu/facpub/234 


\title{
Novel perturbation approach for the structure factor of the attractive hard-core Yukawa fluid
}

\author{
Roman Melnyk \\ Institute for Condensed Matter Physics, National Academy of Sciences of Ukraine, Lviv 79011, Ukraine \\ Filip Moučka \\ Faculty of Science, J. B. Purkinje University, 40096 Ustí nad Labem, Czech Republic \\ Ivo Nezbeda \\ Faculty of Science, J. B. Purkinje University, 40096 Ustí nad Labem, Czech Republic and E. Hála \\ Laboratory of Thermodynamics, ICPF, Academy of Sciences, 16502 Prague 6-Suchdol, Czech Republic \\ Andrij Trokhymchuk \\ Institute for Condensed Matter Physics, National Academy of Sciences of Ukraine, Lviv 79011, Ukraine and \\ Department of Chemistry and Biochemistry, Brigham Young University, Provo, Utah 84602, USA
}

(Received 14 February 2007; accepted 5 July 2007; published online 6 September 2007)

\begin{abstract}
A novel perturbation approach for the structure factor $S(k)$ of the Lennard-Jones-type Yukawa fluid with $z=1.8$ is presented. An approach is based on a new reference system, that is, the short-range Yukawa model with $z_{0}>z=1.8$. By choosing for the reference system the value $z_{0}=6$, it is shown that (i) the proposed approach for $S(k)$ performs much better than the traditional hard-sphere reference perturbation method does; (ii) the use of an approximate mean spherical (MSA) description of the reference structure factor provides the results for $S(k)$ that are more accurate as those obtained from the direct MSA computations; and (iii) the results obtained for $S(k)$ tend to reproduce "exact" simulation results when accurate simulation data for the reference system are used. (C) 2007 American Institute of Physics. [DOI: 10.1063/1.2766937]
\end{abstract}

\section{INTRODUCTION}

It was recognized already long time ago that it is the range of intermolecular interactions that primarily determines the properties of fluid systems. ${ }^{1}$ Different types of fluids are thus modeled by different intermolecular potential functions ranging from medium-ranged nonelectrostatic models on one side to models with explicit long-range Coulombic interactions on the other side. Consequently, such a qualitative difference in the models makes any systematic theoretical investigations of the effect of the range of interactions on the properties of fluids rather difficult (or nearly impossible). For this reason, a model system made up of hard spheres with an attractive tail, $-\epsilon \exp (-z r) / r$, known as the Yukawa (Y) potential, has been the subject of intensive studies for several decades. ${ }^{2}$ By varying decay parameter $z$, the range of the interparticle attraction can be easily changed from being extremely short or intermediate up to extremely long range. The $\mathrm{Y}$ potential is thus rather universal and can be considered as a general effective interaction model of condensed matter ${ }^{3}$ and as such appears in a large variety of different physical applications. ${ }^{4}$ Another, not less important feature making the $\mathrm{Y}$ fluid so attractive is that an approximate analytic result for its both structural and thermodynamic properties is available [via Waisman's solution of the Ornstein-Zernike equation using the mean spherical approximation $^{5}$ (MSA)].

The primary quantity defining the structure of manybody systems is the structure factor $S(k){ }^{6}$ In addition to the "exact," i.e., computer simulation and approximate MSA re- sults, a common alternative route toward $S(k)$ has been via a perturbation expansion. ${ }^{7-10}$ Perturbation expansion is a very powerful and, in general, commonly used method both in liquid state theories and applications. Its success or failure inherently depends on the choice of the reference system, i.e., on the decomposition of the total potential into reference and perturbation parts. For a number of reasons, a natural choice for the reference system has been so far exclusively the fluid of hard spheres and differences between the obtained results reflected thus primarily this choice of the reference (and to a lesser extent differences between the used results for the structure of the reference). It is evident that a reference system that would be physically closer to the structure of the investigated system may be expected to provide better results and it is therefore quite natural and legitimate to ask the question whether there is an alternative to the hard-sphere model, i.e., whether a qualitatively different reference system can be found and that can also simultaneously produce more accurate results.

Such a possibility is explored in this paper. As a test case we consider the medium-range Lennard-Jones-type (YLJ) fluid of $z=1.8$ and use the short-range Yukawa (Y0) fluid of $z_{0}=6$ as a reference. It is shown that this choice yields accurate results even in the case when an approximate MSA result is used for the reference structure factor. However, and what is the most important, we show that the proposed approach allows one to improve the final outcome for $S(k)$ by using a more accurate result for the reference system. After summarizing the basic definitions and properties of the $\mathrm{Y}$ 


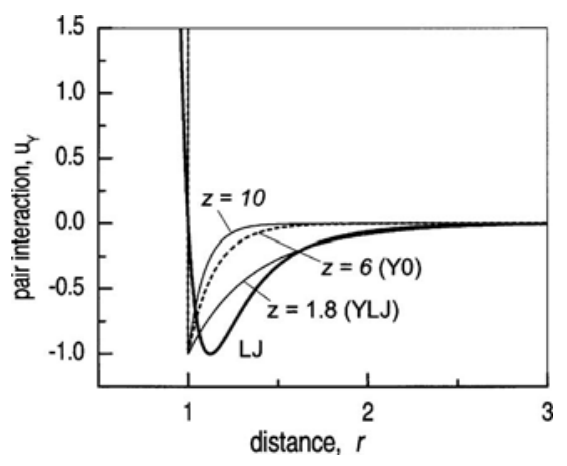

FIG. 1. The Y potential for different values of the $z$ parameter (labels at curves) and its comparison with the Lennard-Jones 6-12 potential.

fluid along with some computational details in the next section, the results obtained for structure factor are presented in Sec. III and discussed then in Sec. IV.

\section{THEORETICAL CONSIDERATION AND COMPUTATIONAL DETAILS}

In general, the Yukawa (Y) intermolecular potential model is defined as follows:

$$
u_{Y}(r)= \begin{cases}\infty, & r<\sigma \\ -\epsilon \sigma \exp [-z(r-\sigma)] / r, & r \geqslant \sigma,\end{cases}
$$

where $\sigma$ is the diameter of the hard core, $\epsilon$ is the well depth, and $z$ is a decay parameter that measures the range of attraction. For simplicity, $\sigma$ and $\epsilon$ are set to unity henceforth. By varying parameter $z$, the range of the interparticle attraction can be easily changed from being extremely short (like the Baxter adhesion when $z \rightarrow \infty$ ) to intermediate (like the Lennard-Jones interaction when $z \sim 1.8$ ) or to extremely long range (like the electrostatic Coulomb interaction when $z \rightarrow 0$ ) (see Fig. 1). For its similarity with the Lennard-Jones $(\mathrm{LJ})$ potential, the Y potential with $z=1.8$ has very often been used as a prototype of an analytically solvable model of simple fluids. ${ }^{2}$ Very likely for this reason, the case $z=1.8$ has also been the most frequently studied $\mathrm{Y}$ model and is the subject of the present paper, too. We will refer henceforth to the Y model with $z=1.8$ as the YLJ model/fluid.

Another important feature of the $\mathrm{Y}$ fluid is that with decreasing the range of attraction, i.e., with increasing the range parameter $z$, its critical point temperature decreases toward its triple point temperature. ${ }^{11-14}$ This means that critical events in the system characterized by the range parameter $z$ will always correspond to the supercritical temperatures for the system that is characterized by the larger value of the range parameter, $z_{0}>z$. Additionally, there is enough evidence that when the attractive range becomes, approximately, less than one-sixth of the hard-core diameter (i.e., when $z_{0} \approx 6$ ) the liquid phase in such a Y fluid disappears completely. ${ }^{1-16}$

In general, the static structure factor $S(k)$, which is the main objective of present study, can be calculated as the Fourier transform of the total correlation function. However, this function need not be simply available and the only theoretical analytic results can be obtained via approximate methods. Typically, the MSA (Refs. 17-21) is used. Al- though there are numbers of publications where MSA structure factor of Y fluid is used in various applications (e.g., see Ref. 20), to the best of our knowledge there is no comparison against computer simulation data, i.e., it is not clear how accurate this MSA result for the structure factor of $\mathrm{Y}$ fluid is.

One more common route toward structure factor is via the perturbation theory ${ }^{7-10}$ and is known as the random phase approximation (RPA) equation, ${ }^{6}$

$$
S(k)=\frac{S_{0}(k)}{1+\beta \rho S_{0}(k) \Phi(k)},
$$

where $\rho=N / V$ is the dimensionless number density and $\beta$ $=1 / k_{B} T \equiv 1 / T^{*}$, where $k_{B}$ is the Boltzmann constant and $T$ is the absolute temperature. To derive this expression ${ }^{7-10}$ it is assumed, similarly as in the MSA, that the pair potential $u_{\mathrm{Y}}(r)$ can be decomposed into reference and perturbation parts,

$$
u_{Y}(r)=v_{0}(r)+w(r),
$$

where $v_{0}$ represents the reference system (denoted by subscript 0 henceforth) and $w=u_{\mathrm{Y}}-v_{0}$ is the attractive tail or perturbation. $S_{0}(k)$ in Eq. (2) thus corresponds to the structure factor of the reference system and $\Phi(k)$ is the Fourier transform of the attractive tail,

$$
\Phi(k)=4 \pi \int_{0}^{\infty} w(r) \frac{\sin (k r)}{k r} r^{2} d r .
$$

Except for the high temperature limit where $S(k) \approx S_{0}(k)$, application of Eq. (2) is the subject to certain constrains that decomposition [Eq. (3)] must satisfy. First of all, the tail $w(r)$ must be sufficiently weak, or density $\rho$ must be sufficiently low to ensure that $\beta \rho S_{0}(k) \Phi(k)>-1$ for all values of $k$. The case $k=0$ is a special case because, in accordance with the compressibility equation, ${ }^{6}$ the value of $S(k=0)$ defines the compressibility of the system. Equation

$$
\beta\left(\frac{\partial p}{\partial \rho}\right)_{T} \equiv \frac{1}{S_{0}(0)}+\beta \rho \Phi(0)=0
$$

and its density derivative are therefore usually used to determine the critical point of the systems with long-range interactions. Using similar considerations, the reference system itself must not have the critical point in order to avoid divergency of $S_{0}(k=0)$ and, consequently, not to introduce an artificial divergency of the total $S(k)$. Additionally, the structure factor of the reference system must be easily accessible in order to accomplish the entire computations.

The structure factors $S(k)$ and $S_{0}(k)$ for the $\mathrm{Y}$ and $\mathrm{Y} 0$ models, respectively, were evaluated in the present study using both the approximate MSA solution and exact simulation data. For convenience, an analytic MSA equation that follows from the results of Refs. 17-21 is given in Appendix. To obtain the exact simulation structure factors we used the standard NVT Monte Carlo molecular simulations with $N=2048$ particles in a cubic $(L \times L \times L)$ box. The Yukawa potential [Eq. (1)] was truncated at the half-length of the box, $R_{c}=L / 2$, and shifted to zero at $r=R_{c}$. In the particle displacement Monte Carlo steps, a randomly selected molecule was attempted to move by no more than $l_{\max }$, the value 

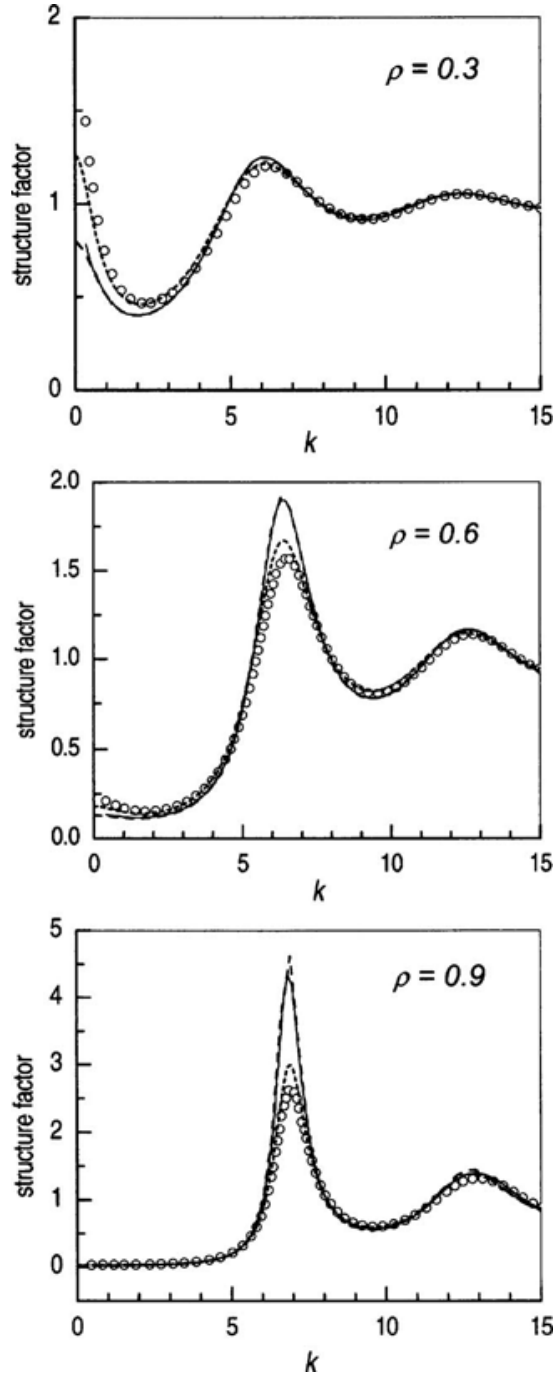

FIG. 2. The structure factor $S(k)$ of the YLJ fluid of $z=1.8$ at the supercritical reduced temperature $T^{*}=1.5$ obtained from simulations (circles) and MSA approximation (short-dashed line), and its comparison with the perturbed HS route using for $S_{0}$ simulation data (full line) and PY result (longdashed line).

of which was set so as to achieve the acceptance ratio about $30 \%$. Approximately $75000 \times N$ displacement attempts were generated to equilibrate the system starting from the fcc initial configuration. In the production runs there were $12 \times N$ displacement attempts made between two consecutive measurements of the structure factor. The averages were determined from about 50000 measurements. For the calculation, integer $\mathbf{k}$ vectors were considered the interval from $(-40,-40,-40)$ to $(40,40,40)$.

\section{A. Hard-sphere reference system}

The simplest nontrivial, and hence also the most frequently used choice for the reference system that satisfies the above conditions is the hard-sphere (HS) system. Thus,

$$
v_{0}(r) \equiv u_{\mathrm{HS}}(r)= \begin{cases}\infty, & r<1 \\ 0, & r \geqslant 1\end{cases}
$$

and

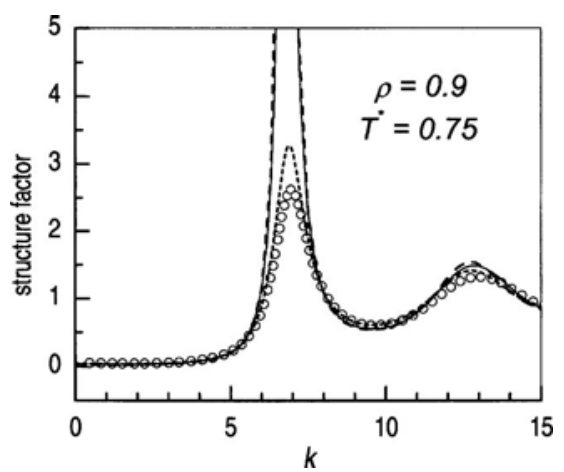

FIG. 3. The same as Fig. 2 for the typical high density liquid state point.

$$
w(r) \equiv w_{\mathrm{Y}-\mathrm{HS}}(r)= \begin{cases}0, & r<1 \\ -\exp [-z(r-1)] / r, & r \geqslant 1,\end{cases}
$$

which yields for $\Phi$ the result

$$
\Phi_{\mathrm{Y}-\mathrm{HS}}(k)=-4 \pi \frac{\cos k+z \sin k / k}{z^{2}+k^{2}} .
$$

We will refer to this level of description as the perturbed HS route.

Figures 2 and 3 show the HS route results for $S(k)$ of the YLJ-fluid and compare them with direct MSA results and the simulation data. We recall that there are two curves resulting from Eq. (2) referring to the way how reference structure factor $S_{0}(k) \equiv S_{\mathrm{HS}}(k)$ was evaluated. One of the curves was obtained by using the Percus-Yevick (PY) approximation while other by using the exact simulation data; in following these two sets of results are referred to as the HS/PY and HS/MC, respectively. First of all, we observe that the accuracy of all approximate approaches is sensitive to the state point of the YLJ fluid. Considering the perturbed HS route, its use can be justified at low densities and high temperatures only regardless whether the approximate PY results (the long-dashed line) or exact simulation data (the solid line) are used for the reference structure factor $S_{0}(k) \equiv S_{\mathrm{HS}}(k)$. In particular, the HS route fails to reproduce correctly two of the

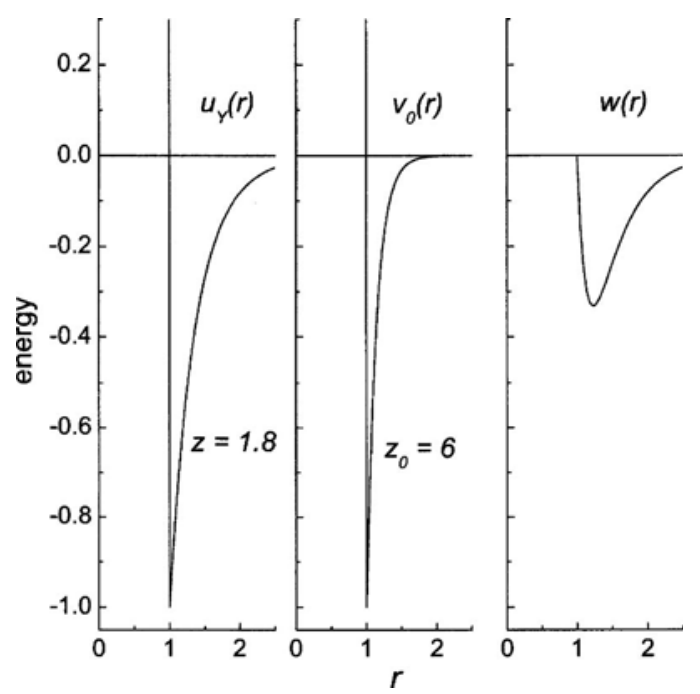

FIG. 4. Decomposition of the total potential $u_{\mathrm{Y}}(r)$ into reference potential $v_{0}(r)$ and long range tail $w(r)$ in accordance with Eqs. (9) and (10). 

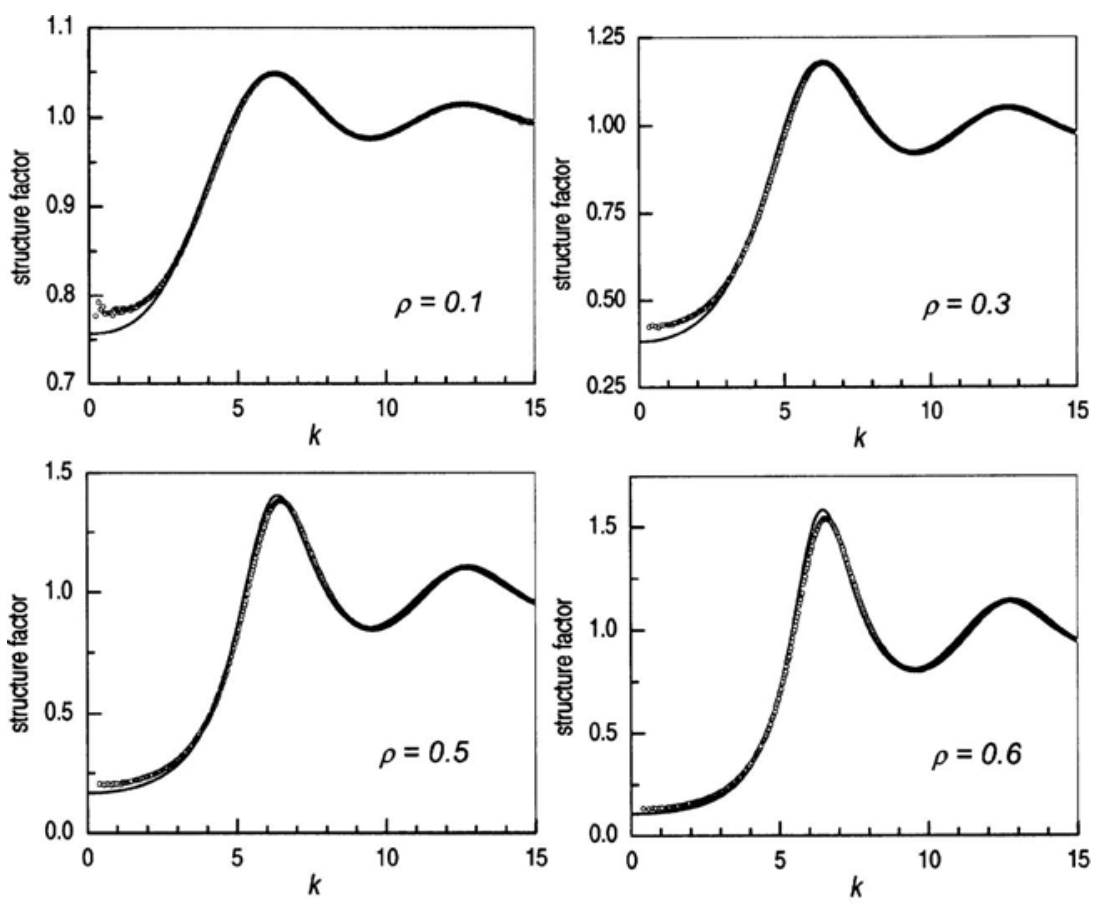

FIG. 5. Structure factors of the shortrange Y0 fluid of $z_{0}=6$ at the reduced temperature $T^{*}=1.5$ obtained from simulations (circles) and the MSA (lines). These structure factors are used as the reference structure factor $S_{0}(k)$ in the calculations via the perturbed $\mathrm{Y} 0$ route.
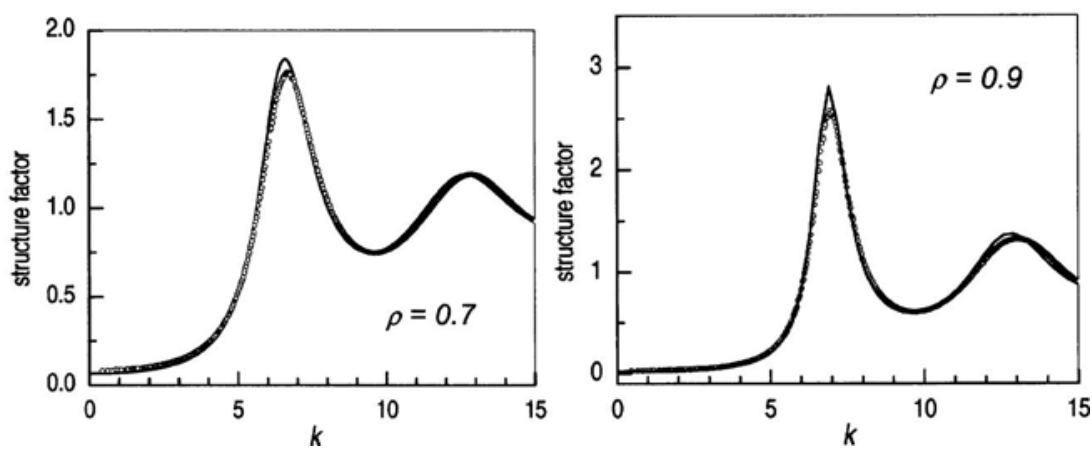

most important features of the YLJ-fluid structure factor, namely, (i) the height of the main peak at intermediate values of $k(k \approx 6)$, and (ii) the behavior of $S(k)$ in the region of low $k$ and at $k=0$. The first feature is related to the liquid/solid transition, while the second concerns the issue of the vapor/ liquid coexistence in the YLJ fluid. At a typical high density liquid state point, the results provided by the perturbed HS route predict an unrealistically high value of the main peak of $S(k)$ and could not be used anymore; the use of the simulation data for $S_{0}(k)$ only slightly improves this failure of the HS route. The direct MSA seems to perform better (see the short-dashed lines). In particular, the height of the main peak of $S(k)$ is considerably closer to the simulation data. Nonetheless, some discrepancies between MSA results and the simulations data around the peaks at larger values of $k$ are still present; similar drawback of the MSA equation holds true for the region of small $k$ including $k=0$.

\section{B. Reference system with a short-range attraction}

The condition of absence of the divergency of $S_{0}(k)$ at $k=0$ along with the availability of an approximate analytical PY results were among the main reasons why the hardsphere structure factor has become widely used as a reference. However, nowadays it appears that the same conditions are satisfied also by the short-range Yukawa fluid, i.e., Y fluid with value of the range parameter $z_{0}$ that is larger then $z$. We will refer henceforth to this fluid as Y0 model. Moreover, comparing with a HS reference system such a Y0 reference system qualitatively is much closer to any system with a long-range attraction. Consequently, one may intuitively expect better convergence of a perturbation expansion about such a Y0 fluid reference. It is thus tempting to write,

$$
v_{0}(r) \equiv u_{\mathrm{Y} 0}(r)= \begin{cases}\infty, & r<1 \\ -\exp \left[-z_{0}(r-1)\right] / r, & r \geqslant 1,\end{cases}
$$

and, consequently,

$$
\begin{aligned}
w(r) & \equiv w_{\mathrm{Y}-\mathrm{Y} 0}(r) \\
& = \begin{cases}0, & r<1 \\
-\left(\exp [-z(r-1)]-\exp \left[-z_{0}(r-1)\right]\right) / r, & r \geqslant 1,\end{cases}
\end{aligned}
$$

which yields for $\Phi$ the result,

$$
\Phi_{\mathrm{Y}-\mathrm{Y} 0}(k)=-4 \pi\left(\frac{\cos k+z \sin k / k}{z^{2}+k^{2}}-\frac{\cos k+z_{0} \sin k / k}{z_{0}^{2}+k^{2}}\right) .
$$

Here $z_{0}>z$ is an appropriately chosen reference decay parameter. In this paper we consider the choice $z_{0}=6$. The cor- 

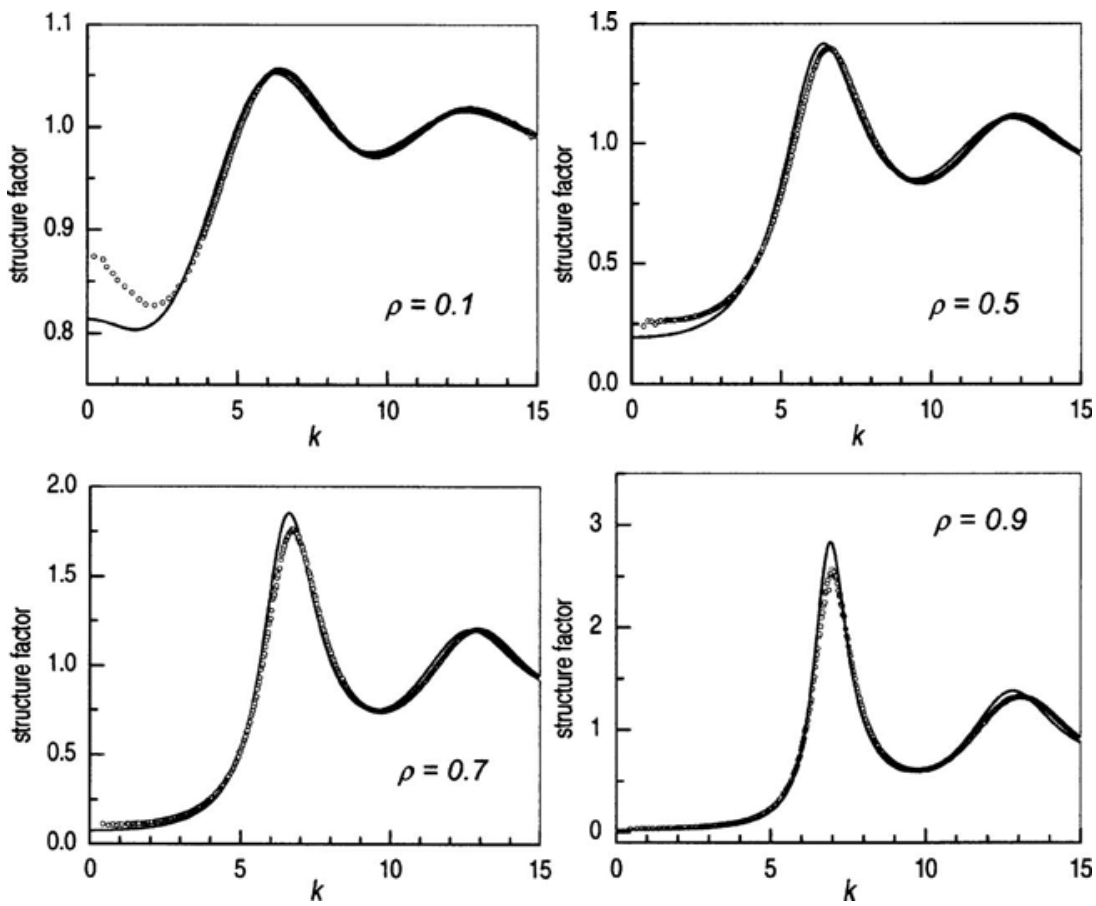

FIG. 6. The same as Fig. 5 for the reduced temperature $T^{*}=1$. responding $u_{\mathrm{Y}}$ potential decomposition is shown in Fig. 4. We will refer henceforth to this level of description as the perturbed Y0 route.

\section{RESULTS AND DISCUSSIONS}

To accomplish the alternative route suggested above requires the structure factor $S_{0}(k) \equiv S_{\mathrm{Y} 0}(k)$ of the novel reference system. The only analytic result available is based on the MSA solution ${ }^{5,17-21}$ and for convenience is given in Appendix. To see what one can expect and how this approximate result for $S_{\mathrm{Y} 0}(k)$ compares with the simulation data we put them together in Figs. 5-7 for a set of thermodynamic states at reduced temperatures $T^{*}=1.5,1$, and 0.75 that correspond to the supercritical, subcritical, and to a typical low temperature state points of the YLJ fluid, respectively. As we can gather from these figures, the MSA theory performs reasonably well in this rather wide temperature range. Systematic noticeable discrepancies are found (i) in the region of small $k$ values at low densities (roughly for $\rho$ smaller than 0.4 ), and (ii) around the peaks at high densities (for $\rho$ greater than, approximately, 0.5) only. It is worth mentioning here that, in general, at high temperatures (approximately for $T^{*}$

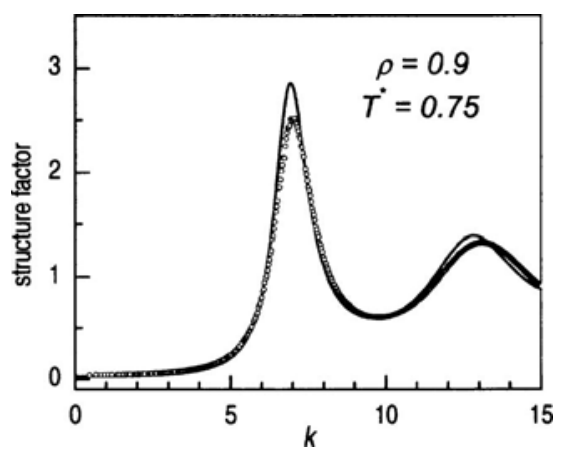

FIG. 7. The same as Fig. 5 for the typical high density liquid state point. greater than 2), the role of the attractive tail gradually diminishes and the MSA result for the Y fluid converges to the PY for the HS fluid; the comparison of the structure factor with simulations in this case is well documented in literature.

The structure factor $S(k)$ of the YLJ fluid that results from the outlined above Y0 route is shown in Figs. 8 and 9. Similar to the HS route discussed in Figs. 2 and 3, there are two curves obtained by using for the reference structure factor $S_{\mathrm{Y} 0}(k)$ either the MSA results (referred to as the $\mathrm{Y} 0 /$ MSA) or the simulation data (referred to as the Y0/MC), respectively. First of all, one can see that in both cases there is a significant improvement over the corresponding results obtained via the HS route. Secondly, we note that the Y0 route based on the MSA for the reference structure factor gives results that are very close to the direct evaluation of $S(k)$ by means of the MSA (see corresponding curves in Figs. 2 and 3). A more careful analysis of the discrepancies around the peaks of $S(k)$ as well as discrepancies at $k \approx 0$ suggests that they could originate from the inaccuracies of the MSA for the reference structure factor $S_{\mathrm{Y} 0}(k)$ (see Figs. 5-7). Indeed, when in Eq. (2) the MSA results for the reference structure is replaced by simulation data, the accuracy of resulting $S(k)$ is improved (solid curves in Figs. 8 and 9). We can see that such a Y0/MC route provides an almost perfect agreement with the simulation data throughout the entire $k$ region.

While the accuracy of the amplitudes of the peaks of $S(k)$ can be tested by a direct comparison with the simulation data, the behavior of $S(k)$ at small $k$ and, especially, the value $S(k=0)$ cannot be due to uncertainty of simulation data in this $k$ region. On the other hand, there is an indirect possibility to examine the value $S(k=0)$ by using Eq. (5) and determine the critical point of the YLJ fluid. Obtained in such a way, the critical density and critical temperature can be compared against the simulation data already available in literature. $^{12-14}$ Whereas for the HS/PY route this is 

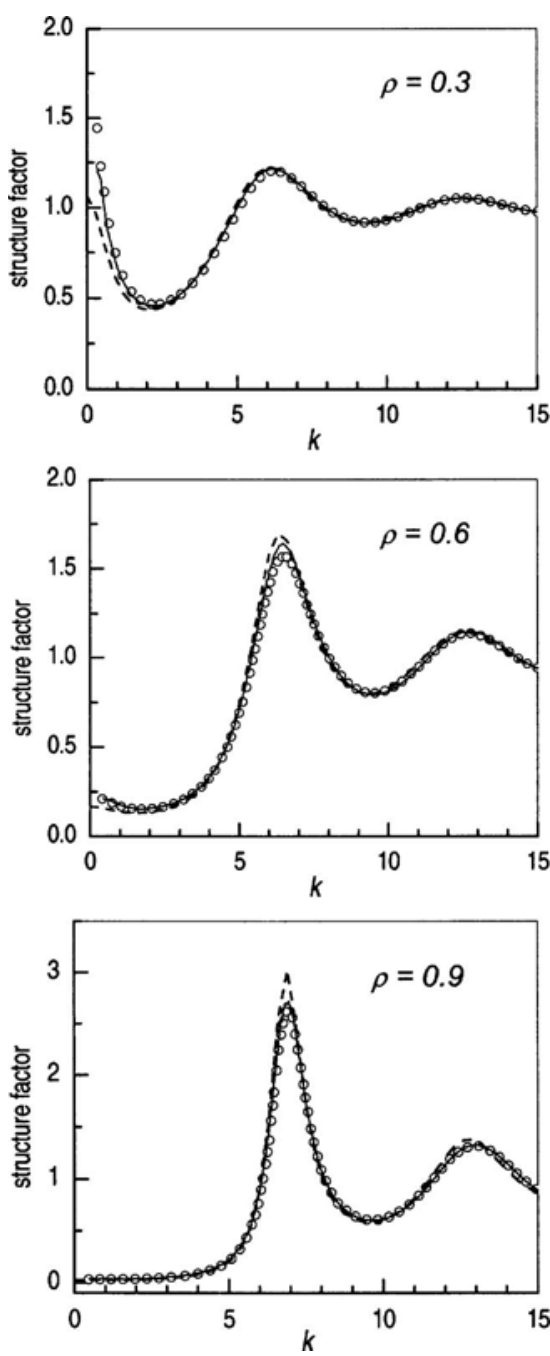

FIG. 8. The structure factor $S(k)$ of the YLJ fluid at the supercritical reduced temperature $T^{*}=1.5$ obtained from simulations (circles) and the perturbed Y0 route [Eqs. (9)-(11)] using the simulation (full line) and MSA (dashed line) for the reference structure factor, $S_{0} \equiv S_{\mathrm{Y} 0}$.

straightforward, ${ }^{6}$ the critical density and temperature of the Y0/MSA approach should be evaluated using the primordial Eq. (5) that in this case has the form

$$
a_{0}^{2}-\frac{24 \eta}{T^{*}}\left(\frac{1+z}{z^{2}}-\frac{1+z_{0}}{z_{0}^{2}}\right)=0
$$

where $a_{0}^{2}$ is the MSA result ${ }^{18,19}$ for the inverse compressibility of the $\mathrm{Y} 0$ reference system characterized by parameter $z_{0}$,

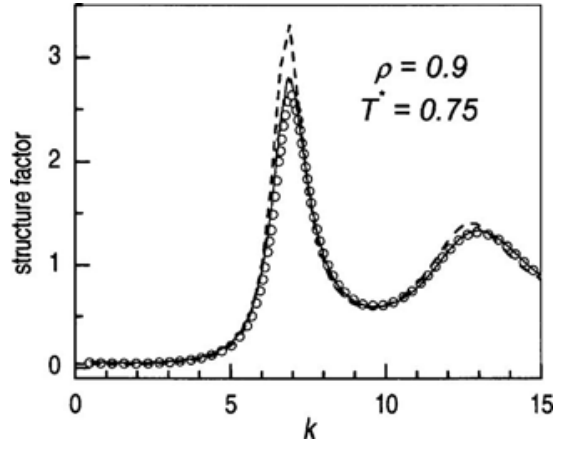

FIG. 9. The same as Fig. 8 for the typical high density liquid state point.

$$
a_{0}=\frac{2 \Gamma}{z_{0}}\left[\frac{\Gamma+z_{0}}{1-\eta}+\frac{1+2 \eta}{(1-\eta)^{2}}\right]+\frac{1+2 \eta}{(1-\eta)^{2}}
$$

with $\eta=(\pi / 6) \rho$ and $\Gamma$ being the scaling parameter of the MSA solution ${ }^{17,18}$ for the Y0 potential. For fixed temperature $T^{*}$, solving Eq. (12) provides two densities, $\rho_{V}$ and $\rho_{L}$, which define the spinodal line for the YLJ fluid. As temperature approaches the critical point temperature $T_{c}^{*}$, there is only one solution for density and corresponds to the critical point density $\rho_{c}$. Within the direct MSA solution such a way to determine the parameters of the critical point is well known as a compressibility route (MSA-C). ${ }^{22,23}$ Available approximate results for the critical point parameters of the YLJ fluid are collected in Table I along with the simulation data. ${ }^{12-14}$ As we can see from this table, the values obtained via the HS route are significantly lower than the simulation results. As expected, the direct MSA performs somewhat better but the critical temperature still remains too low. However, by using the Y0 route based on the MSA description of the reference system one gets parameters of the critical point that are very close to the simulation data.

From other side an estimation of the critical properties may provide us valuable information on the effect of the choice of the reference fluid parameter $z_{0}$ on the final results. In Fig. 10 we show therefore Y0/MSA results for the critical parameters of the YLJ fluid that are obtained with different choices of $z_{0}$. This simple illustration cannot be evidently used for the final assessment regarding the effect of $z_{0}$ but may provide at least a first rough notion. An important technical issue closely related to the choice of the reference $z_{0}$ is an accuracy of the description of the reference system. This effect is illustrated in Fig. 11 where we show three sets of approximate data for the structure factor $S(k)$ of the YLJ fluid at high density $\rho^{*}=0.9$ : two of them are obtained within the perturbed $\mathrm{Y} 0$ route using for the reference fluid the MSA

TABLE I. The critical density and critical temperature of the YLJ fluid of $z=1.8$.

\begin{tabular}{ccccccc}
\hline \hline & HS/PY & MSA-C & Y0/MSA & Simul. $^{\mathrm{b}}$ & Simul. $^{\mathrm{c}}$ & Simul. $^{\mathrm{d}}$ \\
\hline$\rho_{c}$ & 0.245 & 0.32 & 0.281 & 0.308 & 0.294 & 0.313 \\
$T_{c}^{*}$ & 0.972 & 1.010 & 1.082 & 1.179 & 1.192 & 1.177 \\
\hline \hline
\end{tabular}

${ }^{\mathrm{a}}$ References 22 and 23 .

${ }^{\mathrm{b}}$ References 14 .

${ }^{\mathrm{c}}$ References 12 .

${ }^{\mathrm{d}}$ Reference 13 . 


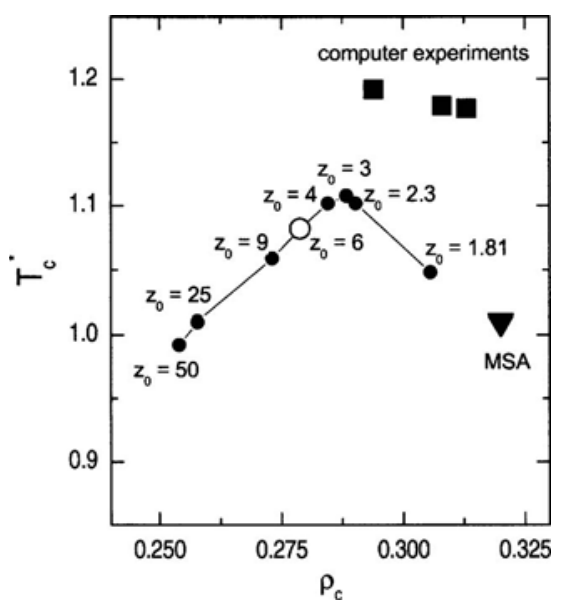

FIG. 10. Dependence of the critical temperature and critical density of the YLJ fluid of $z=1.8$ on the choice of the reference Y0 system parameter $z_{0}$. Filled squares show location of the critical point as predicted by computer simulations (Refs. 12-14) while filled triangle corresponds to the MSA result (Refs. 22 and 23).

and computer simulation; the third one represents then the direct MSA calculation. As we see, with more accurate description of the reference system by the use of the simulation data, brings the calculated structure factor into the agreement with the simulation data throughout the entire $k$ space.

\section{CONCLUSIONS}

We have presented a novel perturbation approach to calculate the structure factor of the medium-range Yukawa (Y) model mimicking a simple Lennard-Jones-type (YLJ) fluid. A by-product of this study is also rather an extensive examination of accuracy of the MSA results for the structure factor of the Y fluid. Although the MSA has been in use for more than four decades we are not aware, to our best knowledge, of any such examination of the MSA predicted structure factor with respect to the simulation data.

A possibility to formulate the perturbation method about a more realistic reference model than the hard sphere reference is based on the effect of the range of attraction on the liquid/vapor coexistence diagram. In particular, within the whole family of attractive Yukawa potentials we distinguish between those that have the critical point and those that do not, referring to them as the long-range and short-range

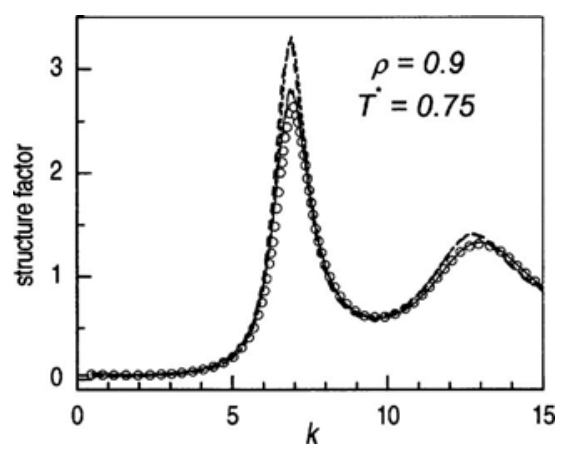

FIG. 11. Comparison of the simulation data (circles) for the structure factor $S(k)$ of the YLJ fluid at a typical high density liquid state point with the direct MSA result (short-dashed line), and the perturbed Y0/MSA (dashed line) and Y0/MC (solid line) results.
Yukawa systems, respectively. Being free from the liquid/ vapor phase transition (or at least exhibiting it below the triple point for the typical simple liquids), the short-range Yukawa (Y0) may play the same role which the fluid of hard spheres has played so far and this possibility has been examined in the present paper.

In this study we have used the short-range Y0 model of $z_{0}=6$ and determined the structure factor of the YLJ fluid using the RPA equation (2). Making use of the analytical solution of the MSA to describe the short-range Yukawa reference structure factor, the calculation procedure, referred to as the perturbed Y0/MSA route, does not exceed in complexity the corresponding HS/PY route calculations based on the PY theory for the hard-sphere structure factor. The same applies to comparison with the direct MSA calculations, providing at the same time also a higher degree of accuracy when compared with computer simulation data.

In the case of the long-wavelength region, where comparison with simulations experiences certain technical difficulties, the accuracy of $S(k=0)$ has been examined by evaluating the parameters of the critical point by means of the compressibility equation (5) and (12). Despite its simplicity, the proposed approach provides results for the critical point of the YLJ fluid that are closest to the simulation data from all the theoretical approaches.

This report should be viewed as a feasibility study to examine the potential of the perturbation method based on a non-hard-sphere reference system. The decomposition of the total pair interaction into the sum of reference and perturbation parts that we used in the present study seems to be rather natural and closer to reality (physical point of view) than other rather mathematical decompositions. The obtained results are clearly very encouraging showing that the appropriate choosing of the short-range Yukawa reference system results in a very accurate description of the YLJ, i.e., LennardJones-type fluid system structure factor within the simple RPA equation. The suggested approach deserves thus a further detailed study including not only physical aspects (e.g., thermodynamic properties) but also technical ones.

\section{ACKNOWLEDGMENTS}

This research was supported by the Czech National Research Program "Information Society" (Project No. 1ET400720409), and by the INTAS Fellowship Grant for Young Scientist (Ref. No. 06-1000019-6184) whose support is also gratefully acknowledged. Authors are thankful to Noe Herrera from Benemérita Universidad Autónoma de Puebla in Mexico for helpful discussions of the MSA equation for the Yukawa fluid structure factor.

\section{APPENDIX: THE MSA RESULT FOR THE STRUCTURE FACTOR OF THE Y FLUID}

Within the MSA, the structure factor $S(k)$ of a onecomponent hard-core Yukawa fluid can be written as, ${ }^{18,20}$

$$
S(k)=\left(\left[1-6 \eta Z_{1}(k)\right]^{2}+36 \eta^{2} Z_{2}^{2}(k)\right)^{-1},
$$

where 


$$
Z_{1}(k)=\frac{1}{k^{2}}\left[(a-\beta)+(a+\beta) \cos (k)-\frac{2 a}{k} \sin (k)\right]+z R(k),
$$

and

$$
\begin{aligned}
Z_{2}(k)= & \frac{1}{k^{2}}\left[\left(k^{2} C e^{-z}+2 a\right) \frac{\cos (k)-1}{k}-\beta k\right. \\
& +(a+\beta) \sin (k)]+k R(k),
\end{aligned}
$$

with

$$
R(k)=\frac{z}{k^{2}+z^{2}}\left[\frac{C+D}{z}-C e^{-z}\left(\frac{\cos (k)}{z}+\frac{\sin (k)}{k}\right)\right] .
$$

All remaining coefficients can be expressed in terms of model parameters, i.e., the packing fraction $\eta=(\pi / 6) \rho$ and $z$, namely,

$$
\begin{aligned}
& a=\frac{2 \Gamma}{z \Delta}\left[\Gamma+z+\frac{1+2 \eta}{\Delta}\right]+\frac{1+2 \eta}{\Delta^{2}}, \\
& \beta=\frac{1}{\Delta}\left[1-\frac{4 \Gamma}{z^{2}}\left(\frac{1+2 \eta}{\Delta}+\frac{z}{2}+\Gamma\right)\right], \\
& D=-\frac{\Gamma}{3 \eta}\left(\Phi_{1} \Gamma+\Phi_{0}\right) e^{z}, \\
& C=-D+\frac{\Gamma}{3 \eta}\left[\frac{\Gamma}{z}\left(1-\alpha_{1}\right)-\frac{\alpha_{0}}{z}\right],
\end{aligned}
$$

where

$$
\begin{aligned}
& \Phi_{1}=\phi_{0}(z)-\frac{12 \eta}{\Delta} \psi_{1}(z), \\
& \Phi_{0}=1+\frac{3 \eta}{\Delta} \phi_{0}(z)-\frac{12 \eta}{\Delta} \psi_{1}(z)\left(1+\frac{z}{2}+\frac{3 \eta}{\Delta}\right), \\
& \alpha_{0}=\alpha_{1}\left(\frac{z}{2}+\frac{1+2 \eta}{\Delta}\right) \\
& \alpha_{1}=\frac{6 \eta}{\Delta z^{2}}[z+2]
\end{aligned}
$$

$$
\phi_{0}(z)=\frac{1}{z}\left(1-e^{-z}\right)
$$

$$
\psi_{1}(z)=\frac{1}{z^{3}}\left[1-\frac{1}{2} z-\left(1+\frac{1}{2} z\right) e^{-z}\right]
$$

and $\Delta=1-\eta$. The MSA parameter $\Gamma$ is defined as the solution of equation,

$$
\Gamma^{2}+z \Gamma=-6 \beta \eta D_{2}(\Gamma),
$$

with

$$
D_{2}(\Gamma)=\left(\frac{1}{\Phi_{1} \Gamma+\Phi_{0}}\right)^{2}
$$

${ }^{1}$ H. C. Andersen, Annu. Rev. Phys. Chem. 26, 145 (1975).

${ }^{2}$ D. Henderson, E. Waisman, J. L. Lebowitz, and L. Blum, Mol. Phys. 35, 241 (1978).

${ }^{3}$ M. H. J. Hagen, E. J. Meijer, G. C. A. M. Mooij, D. Frenkel, and H. N. W. Lekkerkerker, Nature (London) 365, 425 (1993).

${ }^{4}$ J. S. Rowlinson, Physica A 156, 15 (1989).

${ }^{5}$ E. Waisman, Mol. Phys. 25, 45 (1973).

${ }^{6}$ J. P. Hansen and I. R. McDonald, Theory of Simple Liquids, 2nd ed. (Academic, London, 1983).

${ }^{7}$ D. Pines and P. Nozieres, The Theory of Quantum Liquids (Benjamin, New York, 1966).

${ }^{8}$ H. C. Andersen and D. Chandler, J. Chem. Phys. 53, 547 (1970).

${ }^{9}$ H. C. Andersen and D. Chandler, J. Chem. Phys. 55, 1497 (1971).

${ }^{10}$ A. A. Broyles, H. L. Sahlin, and D. D. Carley, Phys. Rev. Lett. 10, 319 (1963).

${ }^{11}$ M. H. J. Hagen and D. Frenkel, J. Chem. Phys. 101, 4093 (1994).

${ }^{12}$ B. Smit and D. Frenkel, Mol. Phys. 74, 35 (1991).

${ }^{13}$ E. Lomba and N. G. Almarza, J. Chem. Phys. 100, 8367 (1994).

${ }^{14}$ M. Gonzalez-Melchor, A. Trokhymchuk, and J. Alejandre, J. Chem. Phys. 115, 3862 (2001).

${ }^{15}$ A. P. Gast, C. K. Hall, and W. B. Russel, J. Colloid Interface Sci. 96, 251 (1983).

${ }^{16}$ H. N. W. Lekkerkerker, W. C.-K. Poon, P. N. Pusey, A. Stroobants, and B. Warre, Europhys. Lett. 20, 559 (1992).

${ }^{17}$ M. Ginoza, J. Phys. Soc. Jpn. 55, 95 (1986).

${ }^{18}$ M. Ginoza, Mol. Phys. 71, 145 (1990).

${ }^{19}$ L. Blum, J. Stat. Phys. 22, 661 (1980).

${ }^{20}$ J. N. Herrera, P. T. Cummings, and H. Ruiz-Estrada, Mol. Phys. 96, 835 (1999).

${ }^{21}$ O. Vazquez and J. N. Herrera, Rev. Mex. Fis. 46, 236 (2000).

${ }^{22}$ P. T. Cummings and E. R. Smith, Chem. Phys. 42, 241 (1979).

${ }^{23}$ D.-M. Duh and L. Mier-y-Teran, Mol. Phys. 90, 373 (1997). 